Pacific Journal of Mathematics

HOMOTOPY PROPERTIES OF LOCALLY COMPACT SPACES
AT INFINITY-CALMNESS AND SMOOTHNESS 


\title{
HOMOTOPY PROPERTIES OF LOCALLY COMPACT SPACES AT INFINITY-CALMNESS AND SMOOTHNESS
}

\author{
ZVONKO ČERIN (ZaGREB)
}

\begin{abstract}
We define two properties of noncompact locally compact spaces called $\mathscr{C}$-calmness at $\infty$ and $(\mathscr{C}, \mathscr{D})$-smoothness at $\infty$ for arbitrary classes of topological spaces $\mathscr{C}$ and $\mathscr{D}$. A number of theorems and examples concerning these properties are given. By considering complements of $Z$-sets in the Hilbert cube from them we get three new shape invariant conditions for compact metric spaces named calmness, $n$-calmness, and $n$-smoothness. Calmness is a movability type condition while $n$-smoothness implies that (and under some additional assumptions is also implied by) the $k$ th shape pro-group of a compactum in question is trivial, for all $k>n$.
\end{abstract}

1. Introduction. This paper continues the study of homotopy properties of noncompact locally compact spaces at $\infty$ from [6], [7], and [8]. In [8] we introduced concepts of calm at $\infty, n$-calm at $\infty$, and $n$-smooth at $\infty$ locally compact spaces. In the present paper these notions are investigated in much the same way as movability at $\infty$ and tameness at $\infty$ were investigated in [6] and [7], respectively. We prove analogous theorems and give a number of examples illustrating those concepts. By a standard $Z$-set complement device [10] (see also [6]) we get three new shape invariant properties of compact metric spaces called calmness, $n$-calmness, and $n$-smoothness. The usefulness of these properties in the future development of shape theory remains to be seen. Our results show that they are rather natural and that one can prove theorems about them resembling some statements about movability and fundamental dimension of compact metric spaces.

We assume the reader is familiar with shape theory of compact metric spaces [2] and with the most elementary concepts and results of infinite dimensional topology [11].

The paper is organized as follows. In $\S 2$ we collect definitions (mostly from [6]) to be used in later sections. The $\S 3$ investigates $\mathscr{C}$-calm at $\infty$ noncompact locally compact spaces, for an arbitrary class of topological spaces $\mathscr{C}$. $\mathscr{C}$-calmness at $\infty$ is a "movability at $\infty$ type" condition for homotopies weaker than the condition SANR $(\infty)$ (or strong movability at $\infty$ ) introduced in the author's thesis [9]. The short $\S 4$ lists properties of calm compact metric 
spaces that follow from results and examples presented in the previous section applying Chapman's $Z$-set complement trick [10]. In $\S 5(\mathscr{C}, \mathscr{D})$-smooth at $\infty$ spaces, where $\mathscr{C}$ and $\mathscr{D}$ are any classes of topological spaces, are considered in a way analoguous to the treatment of $\mathscr{C}$-calm at $\infty$ spaces in $\S 3$. The final $\S 6$ translates these results into shape theory. The class of $n$-smooth compacta obtained in this way is related to the class of those $X$ for which the shape pro-groups $\underline{\pi}_{k}(X)$ are trivial for all $k>n$.

We completed this work during compulsory military service at Military High School in Belgrade. We thank superior officers for providing conditions stimulating research.

2. Notation and definitions. We shall mostly consider noncompact locally compact spaces; two favorite notations for such will be $M$ and $N$. Compact subsets of $M$ and $N$ are denoted with the first few capital Roman letters.

A proper map $f: M \rightarrow N$ is a continuous function such that the preimage under $f$ of every compact subset of $N$ is compact. Proper maps $f, g: M \rightarrow N$ are homotopic at $\infty$ if for every compact $B \subset N$ there is a compact set $A \subset M$ with restrictions $\left.f\right|_{M-A}$ and $\left.g\right|_{M-A}$ homotopic in $N-B$ (notation, $\left.\left.f\right|_{M-A} \simeq g\right|_{M-A}$ in $N-B$ ). We shall say that $M$ homotopy dominates at $\infty$ a space $N$ provided there are proper maps $f: M \rightarrow N$ and $g: N \rightarrow M$ with $f \circ g$ homotopic at $\infty$ to $i d_{N}$, the identity on $N$.

In several situations we shall need to consider inclusions of complements of compact subsets of a space $M$. We denote the inclusion of $M-B$ into $M-A$ by $i_{B, A}$, where $A$ and $B$ are compact subsets of $M$ and $B \supset A$.

Throughout the paper $n \geqq 0$ will be a fixed integer and $\mathscr{C}$ and $\mathscr{D}$, if not stated otherwise, arbitrary nonempty classes of topological spaces. $\mathscr{C} \mathscr{W}$ denotes the class of all $C W$-complexes, $\mathscr{P}$ the class of all finite $C W$-complexes and $\mathscr{P}^{n}$ the subclass of $\mathscr{P}^{n}$ consisting of all complexes of dimension $\leqq n$.

Let $M$ be a space and $U, V, W$, with $V, W \subset U$, its subsets. We let $\mathscr{C}(U, V, W)$ and $\mathscr{C}_{h}(U, V, W)$ denote the following statements.
$\overline{\mathscr{C}(U, V, W)}$ Every $\operatorname{map} f: X \rightarrow V$ of $X \in \mathscr{C}$ is in $U$ homo- topic to a map of $X$ into $W$.
$\overline{\mathscr{C}_{h}(U, V, W)}$ If maps $f, g: X \rightarrow W$ of $X \in \mathscr{C}$ are homotopic in $U$, then they are already homotopic in $V$.

Let $f, g: X \rightarrow Y$ be two maps and $\mathscr{D}$ a class of spaces. We call $f$ and $g \mathscr{D}$-homotopic if for every $Z \in \mathscr{D}$ and a map $h: Z \rightarrow X$, 
the compositions $f \circ h$ and $g \circ h$ are homotopic.

A noncompact locally compact space $M$ is (1) $\mathscr{C}$-trivial at $\infty$, (2) $\mathscr{C}$-movable at $\infty$, (3) $\mathscr{C}$-calm at $\infty$, and $(\mathscr{C}, \mathscr{D})$-smooth at $\infty$ provided

(2.1) for every compact set $A \subset M$ there is a larger compact set $B$ such that every map $f: X \rightarrow M-B$ of $X \in \mathscr{C}$ into a component of $M-B$ is null-homotopic in $M-A$,

(2.2) for every compact set $A \subset M$ there is a compact $B \supset A$ such that for any compact $C \supset A$ the statement $\mathscr{C}(M-A, M-B$, $M-C$ ) holds,

(2.3) for every compact set $A \subset M$ there is a larger compact set $B$ with the property that for any compact $C \supset B$ there is $D \supset C$ making the statement $\mathscr{C}_{h}(M-B, M-C, M-D)$ true, and

(2.4) for every compact set $A \subset M$ there is a compact $B \supset A$ with the property that every two $\mathscr{D}$-homotopic maps $f, g: X \rightarrow$ $M-B$ of $X \in \mathscr{C}$ into $M-B$ are homotopic in $M-A$, is satisfied, respectively.

Spaces $\mathscr{P}$-calm at $\infty$ are called calm at $\infty$, those $\mathscr{P}^{n}$-calm at $\infty$ are called $n$-calm at $\infty$, and spaces $\left(\mathscr{P}, \mathscr{F}^{n}\right)$-smooth at $\infty$ will be called $n$-smooth at $\infty$.

The simplest way of constructing (non compact) locally compact spaces is to look into a complement $M=N-A$ of a closed subset $A$ in a compact space $N$. In handling problems of deciding when $M$ will have a certain homotopy property at $\infty$ it turned out in [6] and [7] that it is useful to assume $A$ satisfies conditions resembling (and implied by) key properties $A$ would have if $A$ were hazy in $N$. This last concept is due to Kozlowski [14] and in case $N$ is an ANR it is equivalent with Anderson's notion of $Z$-sets (see [11]). Our next definition states these conditions.

A closed subset $A$ of a space $X$ is said to be globally right (left) unstable in $X$ if for every open neighborhood $U$ of $A$ in $X$ the inclusion $U-A \hookrightarrow U$ has a right (a left) homotopy inverse.

Finally, in (3.7), (4.8), (5.4), (5.5), and (6.5) we shall use the shape theory of arbitrary topological spaces in the form described by Kozlowski [13].

A class of topological spaces $\mathscr{C}$ shape dominates a class $\mathscr{D}$ provided for every $X \in \mathscr{D}$ there is $Y \in \mathscr{C}$ such that $Y$ shape dominates $X$. In Kozlowski's description this means that there are natural transformations $\mathscr{F}:[X,-] \rightarrow[Y,-]$ and $\mathscr{G}:[Y,-] \rightarrow[X,-]$ between functors $[X,-],[Y,-]: \mathscr{H} \rightarrow$ Sets, where $\mathscr{C}$ is a homotopy category of spaces having the homotopy type of $C W$-complexes, such that $\mathscr{G} \circ \mathscr{F}=\mathscr{F}_{d}$.

3. $\mathscr{C}$-calmness at $\infty$. In this section we shall prove several 
theorems about $\mathscr{C}$-calm at $\infty$ spaces, defined in $\S 2$, and present examples illustrating this concept. Together with examples in $\S 4$ they show that $\mathscr{C}$-calmness at $\infty$ neither implies nor is implied by movability at $\infty$ but that for this rather strong homotopy condition at $\infty$ for a noncompact locally compact space one can prove results similar to those proved for movability at $\infty$ in [6].

(3.1) ExAmple. Let $\sigma=\left\{X_{i}, f_{i}\right\}_{i>0}$ be a direct sequence of compact spaces and let $\operatorname{Map}(\sigma)$ be the infinite mapping cylinder of it obtained by glueing mapping cylinders of maps $f_{i}$ together. One easily sees that $\operatorname{Map}(\sigma)$ is $\mathscr{C}$-calm at $\infty$, for every class $\mathscr{C}$.

(3.2) ExAmple. The product $X \times[0,1)$ of a compact space $X$ with the half-open unit interval can be clearly considered as the infinite mapping cylinder of a direct sequence $\sigma=\left\{X_{i}, f_{i}\right\}_{i>0}$, where $X_{i}=X$ and $f_{i}=i d$ for every $i>0$. Hence, every such product is $\mathscr{C}$-calm at $\infty$, for any class $\mathscr{C}$.

(3.3) Proposition. If a noncompact locally compact, connected, and locally larcwise-connected space $M$ is $\mathscr{C}$-calm at $\infty$, then $M$ has finitely many ends.

Proof. We shall prove that a space $M$ with infinitely many ends can not be $\{X\}$-calm at $\infty$, for any space $X$. Let $B \subset M$ be an arbitrary compact set. If $M-B$ has $n$ components, select a compact $C \supset B$ so that $M-C$ has $(n+1)$ components, and let $D \supset C$ be any compact set. Let $K_{1}$ and $K_{2}$ be two different components of $M-C$ contained in the same component of $M-B$. If $f: X \rightarrow$ $K_{1} \cap(M-D)$ and $g: X \rightarrow K_{2} \cap(M-D)$ are constant maps, then they are homotopic in $M-B$ (since each component of $M-B$ is arcwiseconnected) but are not homotopic in $M-C$. Hence, $\{X\}_{h}(M-B$, $M-C, M-D)$ does not hold.

The above proposition shows that there are many spaces that are not $\mathscr{C}$-calm at $\infty$, regardless of how simple spaces in $\mathscr{C}$ might be. For example, every tree (i.e., a connected, simply connected 1-complex) with infinitely many ends is such a space.

(3.4) EXAMPLE. A noncompact, locally compact, connected, and locally arcwise-connected $\mathscr{C}$-trivial at $\infty$ space with finitely many ends is $\mathscr{C}$-calm at $\infty$.

(3.5) ExAmple. Let $X$ be a closed subset of a locally compact Hausdorff space $Y$ such that the pair $(Y, X)$ satisfies Siebenmann's isotopy compression axiom $I$ - $\operatorname{Comp}(X, Y)[16]$. Then $Y-X$ is $\mathscr{C}$ - 
calm at $\infty$, for any class $\mathscr{C}$.

(3.6) THEOREM. Suppose $M$ homotopy dominates at $\infty$ a space $N$. If $M$ is $\mathscr{C}$-calm at $\infty$, then $N$ is also $\mathscr{C}$-calm at $\infty$.

Proof. Take proper maps $f: M \rightarrow N$ and $g: N \rightarrow M$ such that $f \circ g$ is homotopic at $\infty$ to $i d_{N}$. Let $A \subset N$ be any compact set. Its preimage $A^{\prime}=f^{-1}(A)$ is a compact subset of $M$. As $M$ is $\mathscr{C}$-calm at $\infty$, there is a compact $B^{\prime} \supset A^{\prime}$ satisfying (2.3). Put $B=A \cup$ $g^{-1}\left(B^{\prime}\right)$. Suppose $C \supset B$ is any compact set in $N$. Let $C^{*} \supset C$ have the property that $\left.f \circ g\right|_{N-C^{*}} \simeq i_{C^{*}, C}$ in $N-C$. Let $C^{\prime}=B^{\prime} \cup f^{-1}\left(C^{*}\right)$ and let $D^{\prime}$ be as in (2.3) with respect to $B^{\prime}$ and $C^{\prime}$. Put $D=C \cup$ $g^{-1}\left(D^{\prime}\right)$. Assume $\varphi, \psi: X \rightarrow N-D$ are maps of $X \in \mathscr{C}$ into $N-D$ homotopic in $N-B$. Maps $g \circ \varphi, g \circ \psi: X \rightarrow M-D^{\prime}$ are homotopic in $M-B^{\prime}$. The choice of $B^{\prime}$ and $D^{\prime}$ implies they are homotopic in $M-C^{\prime}$. Hence $f \circ g \circ \varphi$ and $f \circ g \circ \psi$ are homotopic in $N-C^{*}$. But $f \circ g \circ \varphi \simeq \varphi$ in $N-C$ and $f \circ g \circ \psi \simeq \psi$ in $N-C$. So $\varphi$ and $\psi$ are homotopic in $N-C$.

Our next theorem shows that the question of deciding when a locally compact ANR is $\mathscr{C}$-calm at $\infty$ depends only on the shape properties of spaces in $\mathscr{C}$.

(3.7) THEOREM. Let an ANR $M$ be C -calm at $\infty$ and assume a class $\mathscr{D}$ is shape dominated by a class $\mathscr{C}$. Then $M$ is $\mathscr{D}$-calm at $\infty$.

Proof. If $A \subset M$ is any compact subset let $B$ be chosen using the fact that $M$ is $\mathscr{C}$-calm at $\infty$. Now, for any compact $C \supset B$ take $D \supset C$ such that the statement $\mathscr{C}_{h}(M-B, M-C, M-D)$ is true.

Consider an $X \in \mathscr{D}$ and maps $\varphi, \psi: X \rightarrow M-D$ homotopic in $M-B$. As $\mathscr{D}$ is shape dominated by $\mathscr{C}$, there is $Y \in \mathscr{C}$ and natural transformations $\mathscr{F}:[X,-] \rightarrow[Y,-]$ and $\mathscr{G}:[Y,-] \rightarrow[X,-]$ such that $\mathscr{G} \circ \mathscr{F}=\mathscr{F} d$. Observe that representatives $\phi^{\prime}$ and $\psi^{\prime}$ of $\mathscr{F}_{M-D}([\varphi])$ and $\mathscr{F}_{M-D}([\psi])$, respectively, are maps of $Y$ into $M-D$ that are homotopic in $M-B$ because the equality $\left[i_{D, B} \circ \varphi\right]=\left[i_{D, B} \circ \psi\right]$ implies $\left[i_{D, B} \circ \varphi^{\prime}\right]=\left(i_{D, B}\right)_{\#} \circ \mathscr{F}_{M-D}([\varphi])=\mathscr{F}_{M-B}\left(\left[i_{D, B} \circ \varphi\right]\right)=\mathscr{F}_{M-B}\left(\left[i_{D, B} \circ \psi\right]\right)=$ $\left(i_{D, B}\right)_{\sharp} \circ \mathscr{F}_{M-D}([\psi])=\left[i_{D, B} \circ \psi^{\prime}\right]$. By assumption, they are homotopic in $M-C$. But, then $\left(i_{D, C}\right)_{\sharp} \circ \mathscr{G}_{M-D} \circ \mathscr{F}_{M-D}([\varphi])=\left(i_{D, C}\right)_{\sharp} \circ \mathscr{G}_{M-D} \circ \mathscr{F}_{M-D}([\psi])$. Hence, $\left(i_{D, C}\right)_{\sharp}([\varphi])=\left(i_{D, C}\right)_{\sharp}[[\psi])$, which is another way of saying that $\varphi$ and $\psi$ are homotopic in $M-C$.

(3.8) Proposition. If $M$ is $(\mathscr{C}, \mathscr{D})$-smooth at $\infty$ and $\mathscr{D}$-calm at $\infty$, then $M$ is $\mathscr{C}$-calm at $\infty$. 
Proof. Let $A \subset M$ be a compact subset. Select a compact $B \supset$ $A$ using $\mathscr{D}$-calmness at $\infty$ of $M$. If $C$ is any compact set containing $B$, let $C_{1}$ be taken with respect to $C$ applying $(\mathscr{C}, \mathscr{D})$-smoothness at $\infty$ of $M$. Then pick a compact $D \supset C_{1} \supset C$ so that every two maps $\varphi^{\prime}, \psi^{\prime}: Y \rightarrow M-D$ of an $Y \in \mathscr{D}$ into $M-D$ homotopic in $M-B$ are already homotopic in $M-C_{1}$.

Let $\varphi, \psi: X \rightarrow M-D$ be maps of an $X \in \mathscr{C}$ into $M-D$ and assume $\varphi \simeq \psi$ in $M-B$. Clearly, for every space $Y \in \mathscr{D}$ and a $\operatorname{map} \alpha: Y \rightarrow X$ we have $\varphi \circ \alpha \simeq \psi \circ \alpha$ in $M-C_{1}$ so that, by the choice of $C_{1}$, it follows $\varphi \simeq \psi$ in $M-C$.

(3.9) CoRollary. If $M$ is an n-smooth at $\infty$ and $n$-calm at $\infty$ space, then $M$ is calm at $\infty$.

The class of $\mathscr{C}$-calm at $\infty$ spaces behaves well only under the formation of finite "complemented products" as the following theorem and the example (3.12) below show. We first state a technical definition.

(3.10) Definition. A closed subset $A$ of a space $X$ is strongly globally right (left) unstable in $X$ if for each triple $(U, V, W)$, $W \subset V \subset U$, of neighborhoods of $A$ in $X$ there is a map $f:(U, V$, $W) \rightarrow(U-A, V-A, W-A)$ such that $\left.i \circ f\right|_{V} \simeq i d_{V}$ and $i \circ f \simeq$ $i d_{U}\left(\left.f \circ i\right|_{V-A} \simeq i d_{V-A}\right.$ and $\left.f \circ i \simeq i d_{U-A}\right)$, where $i: U-A \hookrightarrow U$ is the inclusion.

Note that a $Z$-set $A$ in the Hilbert cube $Q$ is both right and left strongly globally unstable in $Q$.

(3.11) Theorem. Let $N_{1}, \cdots, N_{n}$ be compact spaces and $X_{1} \subset N_{1}$, $\cdots, X_{n} \subset N_{n}$ strongly globally right unstable subsets. If $X=$ $\prod_{i=1}^{n} X_{i}$ is globally left unstable in $N=\prod_{i=1}^{n} N_{i}$ and each $M_{i}=N_{i}$ $X_{i}(i=1, \cdots, n)$ is $\mathscr{C}$-calm at $\infty$, then $M=N-X$ is also $\mathscr{C}$-calm at $\infty$.

Proof. Let $A \subset M$ be a compact subset of $M$. Pick an open neighborhood $U_{i}$ of $X_{i}$ in $N_{i}(i=1, \cdots, n)$ such that $A \subset N-$ $\prod_{i=1}^{n} U_{i}$. As $M_{i}$ is $\mathscr{C}$-calm at $\infty$, there is a compact set $B_{i} \supset A_{i}=$ $N_{i}-U_{i}$ in $M_{i}$ satisfying (2.3). Put $B=N-\prod_{i=1}^{n}\left(N_{i}-B_{i}\right)$. Assume $C \supset B$ is an arbitrary compact subset of $M$. Let $V_{i} \subset N_{i}-B_{i}$ be an open neighborhood of $X_{i}$ in $N_{i}$ such that $C \subset N-\prod_{i=1}^{n} V_{i}$. For each $C_{i}=N_{i}-V_{i}$ select a compact $D_{i}$ in $M_{i}$ with respect to $B_{i}$ using $\mathscr{C}$-calmness at $\infty$ of $M_{i}$. The compact set $D=N-$ $\prod_{i=1}^{n}\left(N_{i}-D_{i}\right)$ makes the statement $\mathscr{C}_{h}(M-B, M-C, M-D)$ true. Indeed, assume $\varphi, \psi: K \rightarrow M-D$ are maps of $K \in \mathscr{C}$ into $M-$ 
$D$ homotopic in $M-B$. Projections $\pi_{i} \circ \varphi, \pi_{i} \circ \psi: K \rightarrow N_{i}-D_{i}$ are homotopic in $N_{i}-B_{i}(i=1, \cdots, n)$. The assumption that $X_{i}$ is strongly globally right unstable in $N_{i}$ implies there is a map $f_{i}$ : $\left(N_{i}-B_{i}, N_{i}-C_{i}, N_{i}-D_{i}\right) \rightarrow\left(M_{i}-B_{i}, M_{i}-C_{i}, M_{i}-D_{i}\right)$ such that $j_{i} \circ f_{i} \simeq i d_{N_{i}-B_{i}}$ and $\left.k_{i} \circ f_{i}\right|_{N_{i}-C_{i}} \simeq i d_{N_{i}-C_{i}}$, where $j_{i}: M_{i}-B_{i} \hookrightarrow N_{i}-B_{i}$ and $k_{i}: M_{i}-C_{i} \hookrightarrow N_{i}-C_{i}$ are inclusions. Thus, $f_{i} \circ \pi_{i} \circ \varphi, f_{i} \circ \pi_{i} \circ \psi$ : $K \rightarrow M_{i}-D_{i}$ are homotopic in $M_{i}-B_{i}$. The choice of $B_{i}$ and $D_{i}$ gives that they are homotopic in $M_{i}-C_{i}$. But, we know that $\left.k_{i} \circ f_{i}\right|_{N_{i}-C_{i}} \simeq i d_{N_{i}-C_{i}}$ so we get $\pi_{i} \circ \varphi \simeq \pi_{i} \circ \psi$ in $N_{i}-C_{i}$. Hence, $\varphi$ and $\psi$ are homotopic in $\prod_{i=1}^{n}\left(N_{i}-C_{i}\right)$. Now, use left global unstability of $X$ in $N$ to conclude $\varphi \simeq \psi$ in $M-C=(N-C)-X$.

(3.12) EXAMPLE. The following example shows that (3.11) need not be true for a product of infinitely many spaces.

Consider the Hilbert cube $Q$ as the product $B^{2} \times B^{3} \times B^{4} \times \cdots$ of finite-dimensional cells and regard $B^{n}$ as a cone $S^{n-1} \times[0,1] / S^{n-1} \times$ $\{1\}$ over the $(n-1)$-dimensional sphere $S^{n-1}$. Observe that $\widehat{S}^{n-1}=$ $S^{n-1} \times\{0\}$ is a $Z$-set in $B^{n}$. Hence, $X=\prod_{k>1} \hat{S}^{k-1}$ is a $Z$-set in $Q$. We claim that $M=Q-X$ is not $\left\{S^{1}, S^{2}, S^{3}, \cdots\right\}$-calm at $\infty$ even though each $B^{k}-\widehat{S}^{k-1}$ is $\mathscr{C}$-calm at $\infty$, for any class $\mathscr{C}$.

Indeed, given an arbitrary compact subset $B$ of $M$ we see that there is an integer $b$ and real numbers $t_{1}, \cdots, t_{b} \in(0,1)$ such that $B$ is contained in the complement (with respect to $Q$ ) of the set $U=$ $S^{1} \times\left[0, t_{1}\right) \times \cdots \times S^{b} \times\left[0, t_{b}\right) \times B^{b+2} \times B^{b+3} \times \cdots$. Let $C$ be the complement of $V=S^{1} \times\left[0, t_{1}\right) \times \cdots \times S^{b} \times\left[0, t_{b}\right) \times S^{b+1} \times[0,1 / 2) \times$ $B^{b+3} \times B^{b+4} \times \cdots$. Assume $D \supset C$ is a compact set in $M$. Again, we can find an index $d>b$ and numbers $t_{1}^{\prime}<t_{1}, \cdots, t_{b}^{\prime}<t_{b}, t_{b+1}^{\prime}<1 / 2$, $t_{b+2}^{\prime}, \cdots, t_{d}^{\prime}$ in $(0,1)$ such that $D$ is contained in the complement of the set $W=S^{1} \times\left[0, t_{1}^{\prime}\right) \times \cdots \times S^{d} \times\left[0, t_{d}^{\prime}\right) \times B^{d+2} \times B^{d+3} \times \cdots$.

Let $\varphi, \psi: S^{b+1} \rightarrow W$ be maps of $S^{b+1}$ onto the $(2 b+1)$ th coordinate factor of $W$ that are not homotopic. These two maps are homotopic in $U$ since in the $(2 b+1)$ th direction $U$ has $B^{b+2}$ as a factor. Clearly, $\varphi$ and $\psi$ are not homotopic in $V$.

(3.13) ExAMPLE. It follows from Example (4.3) and Theorem (4.19) in [6] that the space $M$ from the previous example is $\mathscr{C}$ movable at $\infty$, for all $\mathscr{C}$. Hence, a $\mathscr{C}$-movable at $\infty$ noncompact locally compact space is not necessarilly $\mathscr{C}$-calm at $\infty$. Now we shall present an example of an $\left\{S^{1}\right\}$-calm at $\infty$ space that is not $\left\{S^{1}\right\}$-movable at $\infty$, where $S^{1}$ is the 1 -sphere.

Let $\sigma=\left\{S_{i}, f_{i}\right\}_{i>0}$ be the inverse sequence with each bonding space $S_{i}$ a copy of $S^{1}$ and with each bonding map $f_{i}$ a map of degree $k_{i}>1$. Let $M=\operatorname{Map}(\sigma)$ be the infinite mapping cylinder of $\sigma$ (see [12]). As $\lim \sigma$ is a $Z$-set in the $A N R \operatorname{Map}(\sigma) \cup \lim \sigma$ [12] 
and is homeomorphic to some nontrivial solenoid, and the later is not movable [2], it follows from Theorem (4.2) in [6] that $M$ is not $\left\{S^{1}\right\}$-movable at $\infty$.

We shall prove that $M$ is $\left\{S^{1}\right\}$-calm at $\infty$. In order to do this, let us first introduce notation for certain subsets of $M$. For any real number $r \geqq 2$, let $M_{r}$ denote the compact subset of $M$ composed of the mapping cylinders (glued together) of the first $j=[r]$ (the greatest integer less than $r$ ) spaces plus the portion of the mapping cylinder of $f_{j}: S_{j+1} \rightarrow S_{j}$ between the $\{0\}$ th level and the $\{r-[r]\}$ th level (in this notation the base $S_{j} \subset \operatorname{Map}\left(f_{j}\right)$ is the $\{0\}$ th level).

Assume $A \subset M$ is an arbitrary compact subset of $M$. Select an integer $a \geqq 2$ so that $A \subset M_{a+(1 / 2)}$, and let $B=M_{a+1}$. If $C \supset B$ is any compact set, pick an integer $c \geqq a+1$ with the property that $M_{c+(1 / 2)}$ contains $C$. Put $D=M_{c+1}$.

Consider any two maps $\varphi, \psi: S^{1} \rightarrow M-D$ homotopic in $M-B$. Since $S^{1}$ is compact, there is an integer $k \geqq c+1$ such that $\varphi\left(S^{1}\right) \cup$ $\psi\left(S^{1}\right) \subset \bar{M}_{k}-M_{c+1}$. By collapsing this last space in a natural way onto $S_{c+1}$, we see that $\varphi$ and $\psi$ are in $\bar{M}_{k}-M_{c+1}$ homotopic to some maps $\varphi^{\prime}$ and $\psi^{\prime}$ of $S^{\prime}$ into $S_{c+1}$, respectively. As $\varphi^{\prime}$ and $\psi^{\prime}$ are homotopic in $M-B$, this time by collapsing onto $S_{a+1}$, we see that

$$
k_{c} \cdot \cdots \cdot k_{a+1} \cdot \operatorname{deg} \varphi^{\prime}=k_{c} \cdot \cdots \cdot k_{a+1} \cdot \operatorname{deg} \psi^{\prime} .
$$

Hence, $\operatorname{deg} \varphi^{\prime}=\operatorname{deg} \psi^{\prime}$ and, therefore, $\varphi^{\prime} \simeq \psi^{\prime}$ in $S_{c+1}$. This clearly implies $\varphi \simeq \psi$ in $M-C$.

The proof of the following proposition is left to the reader.

(3.14) Proposition. If $M$ is $\mathscr{C}$-calm at $\infty$, then each noncompact component of $M$ is $\mathscr{C}$-calm at $\infty$. Conversely, if $M$ has finitely many noncompact components and each of them is $\mathscr{C}$-calm at $\infty$, then $M$ is $\mathscr{C}$-calm at $\infty$.

(3.15) Definition ([6]). Let $M$ be a noncompact locally compact space and let $M_{0} \subset M$ be an open subset. We shall say that $M$ is isotopable into $M_{0}$ if for every compact $B \subset M_{0}$ and every compact $D \supset B$ in $M$, there is an invertible isotopy $h_{t}: M \rightarrow M(0 \leqq t \leqq 1)$ such that $h_{1}(D) \subset M_{0}$ and $\left.h_{t}\right|_{B}=i d$, for all $t$.

(3.16) THEOREM. Let $\mathscr{C}$ be a class of compact spaces. If a locally compact space $M$ is the union of an increasing sequence of its $\mathscr{C}$-calm at $\infty$ open subsets $M_{i}, i \geqq 0$, such that $M_{i+1}$ is isotopable into $M_{i}$, for every $i \geqq 0$, then $M$ is $\mathscr{C}$-calm at $\infty$.

Proof. Let $A \subset M$ be any compact subset. We can assume 
$A \subset M_{0}$. Since $M_{0}$ is $\mathscr{C}$-calm at $\infty$, there is a compact subset $B \supset A$ of $M_{0}$ with the property that for every compact $C^{\prime} \supset B$ in $M_{0}$ there is $D^{\prime} \supset C^{\prime}$ in $M_{0}$ so that every two maps $\varphi^{\prime}, \psi^{\prime}: X \rightarrow M_{0}-D^{\prime}$ of $X \in \mathscr{C}$, homotopic in $M_{0}-B$, are (already) homotopic in $M_{0}-C^{\prime}$.

Consider an arbitrary compact subset $C$ of $M$ containing $B$. Take $n \geqq 0$ with $C \subset M_{n}$. Let $h_{t}: M_{n} \rightarrow M_{n}$ be an invertible isotopy that throws $C$ into $M_{0}$ and keeps $B$ pointwise fixed. Pick $D^{\prime}$ in $M_{0}$ with respect to $B$ and $h_{1}(C)$. Put $D=h_{1}^{-1}\left(D^{\prime}\right)$.

Assume maps $\varphi, \psi: X \rightarrow M-D$ of $X \in \mathscr{C}$ into $M-D$ are homotopic in $M-B$ (via a homotopy $g_{t}: X \rightarrow M-B$ ). Select $m$ such that $\mathrm{U}_{1 \geqq t \geqq 0} g_{t}(X) \subset M_{m}$. If $m>n$, then by applying an invertible isotopy of $M_{m}$ onto itself that is fixed on $D$ and its final stage moves $D \cup\left(\bigcup_{0 \leqq t \leqq 1} g_{t}(X)\right)$ into $M_{n}$, we see that $\phi$ and $\psi$ can be assumed homotopic in $M_{n}-D$. Hence, without loss of generality we can take $m=n$. Now, $h_{1} \circ \varphi, h_{1} \circ \psi: X \rightarrow M_{0}-D^{\prime}$ are homotopic in $M_{0}-B$ (by the same argument) so that $h_{1} \circ \varphi$ and $h_{1} \circ \psi$ are homotopic in $M_{0}-h_{1}(C)$. But then $\varphi$ and $\psi$ are homotopic in $h_{1}^{-1}\left(M_{0}\right.$ $\left.h_{1}(C)\right) \subset M-C$.

The final result in this section shows that ends (see [1] for an enjoyable introduction into Freudenthal's theory of ends) of a $\mathscr{C}$ calm at $\infty$ space satisfy a condition defined in (3.17). The Example (3.19) rules out its converse in general.

(3.17) Definition. An end $e$ of a locally compact space $M$ is $\mathscr{C}$ calm if for every open neighborhood $U$ of $e$ in $F M$, the Freudenthal compactification of $M$, there is another $V \subset U$ such that for every $U^{\prime} \subset V$ there is $V^{\prime} \subset U^{\prime}$ making $\mathscr{C}_{h}\left(V \cap M, U^{\prime} \cap M, V^{\prime} \cap M\right)$ a true statement.

(3.18) THEOREM. If a locally compact space $M$ is $\mathscr{C}$-calm at $\infty$, then every end $e$ of $M$ is $\mathscr{C}$-calm.

Proof. Let $e \in E M$, where $E M$ denotes the end set of $M$. Suppose $U$ is a neighborhood of $e$ in $F M$. Select a neighborhood $U^{*}$ of $e$ inside $U$ so that $U^{*} \cap E M$ is both an open and closed (in the relative topology of $E M$ ) subset of $E M$. Cover $E M$ with finitely many open sets $U_{1}, \cdots, U_{n}$ such that $U_{1}=U^{*}$ and $U_{1} \cap U_{j}=\varnothing$ whenever $j>1$. Put $A=F M-\bigcup_{i=1}^{n} U_{i}$. Pick a compact $B \supset A$ in $M$ using $\mathscr{C}$-calmness at $\infty$ of $M$. Put $V=U^{*} \cap(F M-B)$. Then $V$ is a neighborhood of $e$ in $F M$.

Consider any open neighborhood $U^{\prime}$ of $e$ in $F M$ contained in $V$. Take a neighborhood $\left(U^{\prime}\right)^{*}$ analogously to the way $U$ was chosen, and take similarly open sets $U_{1}^{\prime}, \cdots, U_{k}^{\prime}$ and put $C=F M-\bigcup_{i=1}^{k} U_{i}^{\prime}$. Select a compact subset $D$ of $M$ such that $\mathscr{C}_{h}(M-B, M-C, M-D)$ 
is true. Put $V^{\prime}=(F M-D) \cap\left(U^{\prime}\right)^{*}$.

Assume $\varphi, \psi: X \rightarrow V^{\prime} \cap M$ are maps of $X \in \mathscr{C}$ into $V^{\prime} \cap M$ homotopic in $V \cap M$. Since $V^{\prime} \cap M \subset M-D$ and $V \cap M \subset M-B$, we see that $\varphi$ and $\psi$ are homotopic in $M-C$. But, clearly, this homotopy must be in $\left(U^{\prime}\right)^{*}$ so that $\varphi$ and $\psi$ are homotopic in $(M-$ $C) \cap\left(U^{\prime}\right)^{*} \subset U^{\prime} \cap M$. In other words, the end $e$ is $\mathscr{C}$-calm.

(3.19) Example. Let $T$ be a tree whose end set $E T$ is homeomorphic to the subset $\{0\} \cup\{1 / n \mid n=1,2,3, \cdots\}$ of the real line. We already observed that $T$ is not $\mathscr{C}$-calm at $\infty$, for any class $\mathscr{C}$. On the other hand, one can easily check that every end $e$ of $T$ is $\mathscr{C}$-calm, for each class $\mathscr{C}$.

4. $\mathscr{C}$-calm compacta. Results of the previous section are here transfered into statements about compacta (=compact metric spaces). This is accomplished using the natural equivalence of the shape theory of compacta considered as $Z$-sets in the Hilbert cube $Q$ with the homotopy theory at $\infty$ of complements of $Z$-sets in $Q$ [6] (see also [10]). Applying that equivalence, from $\mathscr{C}$-calmness at $\infty$ we get a shape invariant property of compacta called $\mathscr{C}$ calmness. The class of calm compacta (i.e., $\mathscr{C}$-calm compacta, where $\mathscr{B}=\mathscr{P}$, the class of all finite polyhedra) includes the class of all FANR's and many of their properties are the same as the corresponding properties of FANR's. There are some differences though since calm compacta need not be movable (Example (4. 11)).

(4.1) Definition. A compact metric space $X$ is $\mathscr{C}$-calm provided there is a $Z$-set $X^{\prime}$ in $Q$ homeomorphic with $X$ such that $M=Q-$ $X^{\prime}$ is $\mathscr{C}$-calm at $\infty$. Compacta $\mathscr{P}$-calm will be called calm and those $\mathscr{P}^{n}$-calm will be called $n$-calm.

In order to make the statement of our first theorem shorter we introduce the following notation. For a closed subset $X$ of a space $N$ we write $X \in \mathscr{C}_{h}(N)$ if for every open neighborhood $U$ of $X$ there is $V \subset U$ such that for every open neighborhood $U^{\prime} \subset V$ we can find smaller $V^{\prime}$ making $\mathscr{C}_{h}\left(V, U^{\prime}, V^{\prime}\right)$ true.

(4.2) THEOREM. The following assertions about a compactum $X$ are equivalent.

(i) $X$ is Co-calm.

(ii) If $X^{\prime}$ is a Z-set copy of $X$ in an ANR $N$, then $N-X^{\prime}$ is $\mathscr{B}$-calm at $\infty$.

(iii) If $X^{\prime}$ is a $Z$-set copy of $X$ in an $A N R N$, then $X^{\prime} \in \mathscr{C}_{h}(N)$.

(iv) If $X$ is a closed subset of an $A N R N$, then $X \in \mathscr{C}_{h}(N)$.

(v) If $X=\lim \sigma$, where $\sigma=\left\{X_{i}, f_{i^{\prime}}^{i}\right\}_{i^{\prime} \geqq i>0}$ is an inverse sequence 
of ANR's, then the infinite mapping cylinder Map( $\sigma)$ of $\sigma$ is $\mathscr{C}$ calm at $\infty$.

(vi) Assume $X=\lim \sigma$, where $\sigma=\left\{X_{i}, f_{i^{\prime}}^{i}\right\}_{i^{\prime} \geqq i>0}$ is an inverse sequence of ANR's. Then for every index $i>0$ there is $j \geqq i$ such that for every $i^{\prime} \geqq j$ we can find $j^{\prime} \geqq i^{\prime}$ with the following property. If $\varphi, \psi: K \rightarrow X_{j^{\prime}}$ are maps of $K \in \mathscr{C}$ into $X_{j^{\prime}}$ such that $f_{j^{\prime}}^{j} \circ \varphi$ and $f_{j^{\prime} \circ \psi}^{j}$ are homotopic, then $f_{j^{\prime} \circ \varphi}^{i, \circ}$ and $f_{j^{\prime} \circ}^{i \prime} \psi$ are homotopic.

Proof. (i ) $\Rightarrow$ ( ii ). In [6] we observed that $N-X^{\prime}$ is homotopy equivalent at $\infty$ to a complement of a $Z$-set copy of $X$ in $Q$. Hence, (ii) follows from (i) by Theorem (3.6).

(ii) $\Rightarrow$ (iii). This implication is immediate once we note that if $A \subset M=N-X^{\prime}$ is a compact subset, then $U=N-A$ is an open neighborhood of $X^{\prime}$ in $N$ and the inclusion $M-A \hookrightarrow U$ is a homotopy equivalence.

(iii) $\Rightarrow$ (iv). Consider $N \times Q$ as a $Z$-set in $Q$, and let $Y$ be a $Z$-set copy of $X$ in $Q$. Since $X^{\prime}=X \times Q$ and $Y$ have the same shape, complements $Q-Y$ and $Q-X^{\prime}$ are homeomorphic [10]. Hence, (iii) holds for $X^{\prime}$. But, $N \times Q$ is collared in $Q$, i.e., $N \times Q$ has a neighborhood of the form $N \times Q \times[0,1)$ with the 0 -level corresponding to $N \times Q$ [11]. It is easily proved that this implies $X \times Q \in \mathscr{C}_{h}(N \times Q)$, and hence also $X \in \mathscr{C}_{h}(N)$ because the projection of $N \times Q$ onto $N$ is a homotopy equivalence over every subset of $N$.

(iv) $\Rightarrow(v)$. An easy proof is based on the observation that $\operatorname{Map}(\sigma)$ can be compactified to an ANR $N=\operatorname{Map}(\sigma) \cup \lim \sigma$ by adding $\lim \sigma$ such that $\lim \sigma$ is a $Z$-set in $N[12]$.

$(\mathrm{v}) \Leftrightarrow(\mathrm{vi})$. A simple proof of that equivalence uses the facts that $N=\operatorname{Map}(\sigma) \cup \lim \sigma$ is homotopy equivalent to $X_{1}$ and that

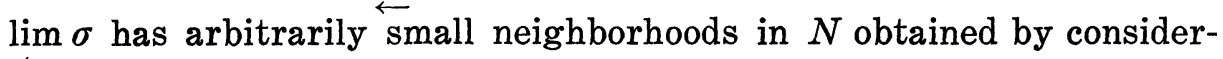
ing infinite mapping cylinders of cofinite subsequences of $\sigma$.

(v) $\Rightarrow$ (i). Suppose $X=\lim \sigma$, where $\sigma=\left\{X_{i}, f_{i^{\prime}}^{i}\right\}_{i^{\prime} \geqq i>0}$ is an inverse sequence of finite polyhedra, and suppose $\operatorname{Map}(\sigma)$ is $\mathscr{C}$-calm at $\infty$. Let $X_{0}$ be the one-point space and let $f_{1}^{0}: X_{1} \rightarrow X_{0}$ be the obvious map. The infinite mapping cylinder $\operatorname{Map}\left(\sigma^{\prime}\right)$ of $\sigma^{\prime}=\left\{X_{i}\right.$, $\left.f_{i^{\prime}}^{i}\right\}_{i^{\prime} \geqq i \geq 0}$ is homotopy equivalent at $\infty$ with $\operatorname{Map}(\sigma)$ and $\lim \sigma^{\prime} \cong \lim \sigma$. Hence, $\operatorname{Map}\left(\sigma^{\prime}\right) \times Q$ is $\mathscr{C}$-calm at $\infty$ (by Theorem $\leftarrow(3.6)$ ). $\overleftarrow{\text { But, }}$ $\left(\operatorname{Map}\left(\sigma^{\prime}\right) \cup \lim \sigma^{\prime}\right) \times Q$ is a contractible compact $Q$-manifold and 
$\left(\lim \sigma^{\prime}\right) \times Q$ is a $Z$-set in it. By [11] that manifold is in fact a Hilbert cube so that $X \times Q$ is $\mathscr{C}$-calm. Applying Theorem (4.7) below, it follows $X$ is $\mathscr{C}$-calm.

(4.3) Corollary. Let $\sigma=\left\{X_{i}, f_{i}\right\}_{i>0}$ be an inverse sequence of ANR's (of dimension $\leqq n)$. A compactum $X=\lim \sigma$ is calm ( $n$ calm) if and only if $X$ is $\left\{X_{1}, X_{2}, \cdots\right\}$-calm.

As there are only countably many homotopy types among compact ANR's (of dimension $\leqq n$ ) [3] in view of (4.3) and (3.7) we immediately get.

(4.4) Corollary. (a) There is a sequence $P_{1}, P_{2}, \cdots$ of finite polyhedra (of dimension $\leqq n$ ) such that a compactum $X$ is calm $\left(n\right.$-calm) if and only if $X$ is $\left\{P_{1}, P_{2}, \cdots\right\}$-calm.

(b) There is a compactum $C$ (of dimension $\leqq n$ ) such that a compactum $X$ is calm (n-calm) if and only if $X$ is $\{C\}$-calm.

Proof. (b) The one-point compactification of the disjoint union of $P_{i}^{\prime}$ s from (a) is one such compactum.

We now list theorems and examples concerning the classes of $\mathscr{C}$-calm and calm compacta that are consequences of results and examples in $\S 3$. The proofs are mostly omitted. The numbers in square brackets denote the result(s) from $\S 3$ implying the statement in question.

(4.5) ExAMPLe ([3.2] and [3.6]). Every FANR compactum $X$ is $\mathscr{C}$-calm, for every class $\mathscr{C}$.

Proof. Let $Y$ be an ANR that shape dominates $X$ and assume both $X$ and $Y$ are $Z$-sets in $Q$. By the Collaring Theorem [11], $(Q-Y) \times Q$ has at infinity the form $Y \times Q \times[0,1)$. Since $(Q-$ $Y) \times Q$ is homotopy equivalent at $\infty$ to $Q-Y$ and $Q-Y$ homotopy dominates at $\infty Q-X,(3.6)$ and (3.2) apply.

For pointed FANR's this also follows from (3.5) because a $Z$ set $X \subset Q$ satisfies the isotopy compression axiom $I$-Comp $(X, Q)$ if and only if $X$ is a pointed FANR [17].

(4.6) Theorem ([3.18] and [3.3]). A compactum $X$ is $\mathscr{C}$-calm if and only if $X$ has finitely many $\mathscr{C}$-calm components.

(4.7) TheOREM ([3.6]). If a compactum $X$ is $\mathscr{C}$-calm and 
$\operatorname{Sh}(X) \geqq S h(Y)$, then a compactum $Y$ is also $\mathscr{C}$-calm.

(4.8) THEOREM ([3.7]). If a compactum $X$ is $\mathscr{C}$-calm and a class $\mathscr{D}$ is shape dominated by a class $\mathscr{C}$, then $X$ is also $\mathscr{D}$-calm.

(4.9) TheOREM ([3.11]). Let $X_{1}, \cdots, X_{n}$ be C-calm compacta. Then their cartesian product $X=\prod_{i=1}^{n} X_{i}$ is $\mathscr{C}$-calm.

(4.10) EXAMPLe ([3.12]). The countable infinite product $S^{\infty}=$ $\Pi_{i>0} S^{i}$ of all finite-dimensional spheres is not calm. Note that $S^{\infty}$ is movable [2].

(4.11) Example ([3.13] and [4.3]). Every solenoid is calm.

Problem (8.2) on the page 266 of Borsuk's book [2] can be phrased in the following form: "If $X=\bigcap_{i>0} X_{i}$ where $X_{1}, X_{2}, \ldots$ are ANR's and $X_{i+1}$ is a retract of $X_{i}$, for every $i=1,2,3, \cdots$, then what shape properties has $X$ ?". The last theorem in this section shows that calmness is one of them.

(4.12) Theorem. If $X_{1}, X_{2}, \cdots$ are $A N R$-sets and if $X_{k+1}$ is a retract of $X_{k}$ for every $k=1,2, \cdots$, then the set $X=\bigcap_{k=1}^{\infty} X_{k}$ is $\mathscr{C}$-calm for every class $\mathscr{C}$.

Proof. We may assume $X_{1} \subset Q$, By using the equivalence of (i) and (iv) of Theorem 4.2 we shall prove that $X$ is calm. This will clearly suffice. Let $U$ be an open neighborhood of $X$ in $Q$. Select $k$ so that $X_{k} \subset U$. Since $X_{k}$ is an ANR, there is an open neighborhood $V$ of $X_{k}$ in $U$ and a retraction $r: V \rightarrow X_{k}$. Let $U^{\prime} \subset$ $V$ be an arbitrary open neighborhood of $X$ in $Q$. Pick $j \geqq k$ so that $X_{j} \subset U^{\prime}$. Let $V^{*}$ be a small open neighborhood of $X_{j}$ in $U^{\prime}$ for which there is a retraction $r^{*}: V^{*} \rightarrow X_{j}$. On a closed subset $T=V^{*} \times\{0,1\} \cup X_{j} \times[0,1]$ of $V^{*} \times[0,1]$ define a map into $V^{*}$ as $r^{*}$ on $V^{*} \times\{0\}$ and as the identity on $V^{*} \times\{1\} \cup X_{j} \times[0,1]$. Since $V^{*}$ is an ANR, there is a neighborhood $N$ of $T$ in $V^{*} \times[0,1]$ and an extension of the above map to all of $N$. Hence, there is an open neighborhood $V^{\prime}$ of $X_{j} \supset X$ such that $V^{\prime} \times[0,1] \subset N$. Thus we can assume that $i d_{V^{\prime}}$ is in $V^{*} \subset U^{\prime}$ homotopic to a retraction $r^{\prime}: V^{\prime} \rightarrow X_{j}$. We claim that the statement $\mathscr{P}_{h}\left(V, U^{\prime}, V^{\prime}\right)$ is true.

Indeed, assume $\varphi, \psi: P \rightarrow V^{\prime}$ are maps of a finite complex $P$ into $V^{\prime}$ and let $g_{t}: P \rightarrow V$ be a homotopy in $V$ between them. Observe that $r^{\prime} \circ \varphi \simeq \varphi$ in $U^{\prime}$ and $r^{\prime} \circ \psi \simeq \psi$ in $U^{\prime}$. Hence maps $r^{\prime} \circ \varphi$ and $r^{\prime} \circ \psi$ are homotopic in $V$ (compose the last two homotopies and $\left.g_{t}\right)$. Let $R: X_{k} \rightarrow X_{j}$ be a retraction. As $r\left(r^{\prime} \circ \varphi\right)=r^{\prime} \circ \varphi$ and $r\left(r^{\prime} \circ \psi\right)=r^{\prime} \circ \psi$ are homotopic in $r(V)=X_{k}$ we see that $R \circ r \circ\left(r^{\prime} \circ \varphi\right)=$ 
$r^{\prime} \circ \varphi$ and $R \circ r \circ\left(r^{\prime} \circ \psi\right)=r^{\prime} \circ \psi$ are homotopic in $X_{j}$. Therefore, $\varphi$ and $\psi$ are homotopic in $U^{\prime}$ and the proof is complete.

5. $(\mathscr{C}, \mathscr{D})$-smoothness at $\infty$. In $\S 2$ we defined, for arbitrary classes $\mathscr{C}$ and $\mathscr{D}$ of topological spaces, the notion of a $(\mathscr{C}, \mathscr{D})$ smooth at $\infty$ noncompact locally compact space, that by Example (5.1) below can be considered as a generalization of a notion of a trivial at $\infty$ space. Here we shall prove a number of theorems and present some examples aimed at giving better understanding of the real meaning of that concept. The most interesting in this direction are results (5.14) and (5.17) where we get a partial characterization of $n$-smooth at $\infty$ spaces.

(5.1) EXAMPLE. A noncompact locally compact locally arcwiseconnected space $M$ is $\left(\mathscr{P}^{\circ}, \mathscr{P}^{\circ}\right)$-smooth at $\infty$ if and only if $M$ is $\mathscr{P}$-trivial at $\infty$.

(5.2) Theorem. If a space $M$ is ( $\mathscr{C}, \mathscr{D})$-smooth at $\infty$ and $M$ homotopy dominates at $\infty$ a space $N$, then $N$ is also $(\mathscr{C}, \mathscr{D})$-smooth at $\infty$.

Proof. Let $f: M \rightarrow N$ and $g: N \rightarrow M$ be proper maps such that $f \circ g$ is homotopic at $\infty$ to $i d_{N}$ and let $A \subset N$ be a compact set. Take a compact $A^{\prime} \supset A$ with $\left.f \circ g\right|_{N-A^{\prime}} \simeq i_{A^{\prime}, A}$ in $N-A$. Let $A^{*}$ be a compact subset of $M$ with the property that $f\left(M-A^{*}\right) \subset N-A^{\prime}$. Select $B^{*} \supset A^{*}$ using the fact that $M$ is $(\mathscr{C}, \mathscr{D})$-smooth at $\infty$. Let $B=g^{-1}\left(B^{*}\right) \cup A^{\prime}$.

Consider $X \in \mathscr{C}$ and any two $\mathscr{D}$-homotopic maps $\varphi$ and $\psi$ of $X$ into $N-B$. Then $g \circ \varphi, g \circ \psi: X \rightarrow M-B^{*}$ are $\mathscr{D}$-homotopic maps. Hence, they are homotopic in $M-A^{*}$. Composing that homotopy with $f$ we see that $f \circ g \circ \varphi$ and $f \circ g \circ \psi$ are homotopic in $N-A^{\prime}$. The choice of $A^{\prime}$ implies $\phi$ and $\psi$ are homotopic in $N-A$.

(5.3) REMARK. In the above theorem the assumption that $M$ homotopy dominates at $\infty N$ can be weakened by assuming that $M$ only quasi-dominates at $\infty N$ [6].

All of the properties at $\infty$ defined in $\S 2$ depend only on shapes of spaces in the class $\mathscr{C}$ when the space under consideration is an ANR. The following two propositions establish this for $(\mathscr{C}, \mathscr{D})$ smoothness at $\infty$ under the additional assumption that spaces in $\mathscr{C}$ have homotopy types of ANR's. Note that for these spaces a shape domination is the same as a homotopy domination. 
types of ANR's. Let a class $\mathscr{D}$ shape dominate a class $\mathscr{D}^{\prime}$. If an ANR $M$ is $\left(\mathscr{C}, \mathscr{D}^{\prime}\right)$-smooth at $\infty$, then $M$ is (CE, $\left.\mathscr{C}\right)$-smooth at $\infty$.

Proof. Let $A$ be a compact set in $M$. Pick a compact $B \supset A$ applying the assumption that $M$ is $\left(\mathscr{C}, \mathscr{D}^{\prime}\right)$-smooth at $\infty$. Let $\varphi$, i: $X \rightarrow M-B$ be maps of $X \in \mathscr{C}$ into $M-B$ with the property that for every space $Y$ in $\mathscr{D}$ and a map $f: Y \rightarrow X$ compositions $\varphi \circ f$ and $\psi \circ f$ are homotopic. We claim that $\varphi$ and $\psi$ are $\mathscr{D}^{\prime}$-homotopic, and therefore homotopic in $M-A$.

Indeed, if $Y^{\prime} \in \mathscr{D}^{\prime}$ take $Y \in \mathscr{D}$ that shape dominates $Y^{\prime}$ and natural transformations $\mathscr{F}:\left[Y^{\prime},-\right] \rightarrow[Y,-]$ and $\mathscr{C}:[Y,-] \rightarrow\left[Y^{\prime}\right.$, - ] such that $\mathscr{G} \circ \mathscr{F}=\mathscr{F} d$ (see $\S 2)$. A homotopy class $\mathscr{F}_{X}\left(\left[f^{\prime}\right]\right) \epsilon$ $[Y, X]$ by assumption satisfies $\varphi_{\sharp}\left(\mathscr{F}_{X}\left(\left[f^{\prime}\right]\right)\right)=\psi_{\sharp}\left(\mathscr{F}_{X}\left(\left[f^{\prime}\right]\right)\right)$, where $f^{\prime}: Y^{\prime} \rightarrow X$ is an arbitrary map. Hence, since $\mathscr{F}$ is natural, $\mathscr{F}_{M-B}\left(\left[\varphi \circ f^{\prime}\right]\right)=\mathscr{F}_{M-B}\left(\left[\psi \circ f^{\prime}\right]\right)$. But then $\mathscr{G}_{M-B} \circ \mathscr{F}_{M-B}\left(\left[\varphi \circ f^{\prime}\right]\right)=\mathscr{G}_{M-B} \circ$ $\mathscr{F}_{M-B}\left(\left[\psi \circ f^{\prime}\right]\right)$ and, finally, $\left[\varphi \circ f^{\prime}\right]=\left[\psi \circ f^{\prime}\right]$ which is another way of saying that $\varphi \circ f^{\prime}$ and $\psi \circ f^{\prime}$ are homotopic, i.e., that $\varphi$ and $\psi$ are $\mathscr{D}^{\prime}$-homotopic.

(5.5) Proposition. Let $\mathscr{C}$ and $\mathscr{C}^{\prime}$ be classes of spaces with homotopy types of polyhedra and assume $\mathscr{C}^{\prime}$ shape dominates $\mathscr{C}$. If an ANR space $M$ is ( $\left.\mathscr{C}^{\prime}, \mathscr{D}\right)$-smooth at $\infty$, then $M$ is also ( $\mathscr{C}$, $\mathscr{D})$-smooth at $\infty$.

Proof. Let $A$ be a compact set in $M$. Pick a compact $B \supset A$ using the fact that $M$ is $\left(\mathscr{C}^{\prime}, \mathscr{D}\right)$-smooth at $\infty$. Let $\varphi, \psi: X \rightarrow$ $M-B$ be $\mathscr{D}$-homotopic maps of $X \in \mathscr{C}$ into $M-B$. Take $X^{\prime} \in \mathscr{C}^{\prime}$ such that $X^{\prime}$ homotopy dominates $X$. Let $\alpha: X \rightarrow X^{\prime}$ and $\beta: X^{\prime} \rightarrow$ $X$ be maps with $\beta \circ \alpha \simeq i d_{X}$.

Consider an arbitrary map $f: Y \rightarrow X^{\prime}$ of $Y \in \mathscr{D}$ into $X^{\prime}$. By assumption, $\varphi \circ \beta \circ f$ and $\psi \circ \beta \circ f$ are homotopic. The choice of $B$ implies $\varphi \circ \beta \simeq \psi \circ \beta$ in $M-A$. Hence, $\varphi \circ \beta \circ \alpha \simeq \psi \circ \beta \circ \alpha$ in $M-A$, and finally $\varphi \simeq \psi$ in $M-A$.

The product of two spaces $(\mathscr{C}, \mathscr{D})$-smooth at $\infty$ need not be $(\mathscr{C}, \mathscr{D})$-smooth at $\infty$ (for example, the real line $R$ is $(\mathscr{P}, \mathscr{P} 0$ )smooth at $\infty$, by (5.1), while the plane $R^{2}=R \times R$ is not, again by (5.1) since $R^{2}$ is not $\mathscr{P}$-trivial at $\infty$ ), but a "complemented product theorem" holds.

(5.6) THEOREM. Let $X_{i}$ be a closed subset of a compact contractible space $N_{i}$, for each $i$ in a set of indices $I$. Put $N=\prod_{i \in I} N_{i}$, $X=\Pi_{i \in I} X_{i}, M=N-X$, and $M_{i}=N_{i}-X_{i}$. If each $M_{i}$ is $(\mathscr{C}, \mathscr{D})-$ smooth at $\infty$, each $X_{i}$ is globally right unstable in $N_{i}$, and $X$ is 
globally left unstable in $N$, then $M$ is $(\mathscr{C}, \mathscr{D})$-smooth at $\infty$.

Proof. Let $A$ be an arbitrary compact subset of $M$. Its complement $N-A$ in $N$ is an open neighborhood of $X$. Hence, there is an integer $n \geqq 1$, indices $i_{1}, \cdots, i_{n}$ in $I$, and compact sets $A_{1} \subset$ $M_{i_{1}}, \cdots, A_{n} \subset M_{i_{n}}$ such that $\left(N_{i_{1}}-A_{1}\right) \times \cdots \times\left(N_{i_{n}}-A_{n}\right) \times \prod_{i \neq i_{1}, \cdots, i_{n}} N_{i} \subset$ $N-A$. Select compact sets $B_{1}, \cdots, B_{n}$ using ( $\left.\mathscr{C}, \mathscr{D}\right)$-smoothness at $\infty$ of the $M_{i_{j}}$ 's. Put $B=\bigcup_{i=1}^{n}\left(B_{i} \times \prod_{j \neq i_{1}, \cdots, i_{n}} N_{j}\right)$.

Consider any space $X \in \mathscr{C}$ and $\mathscr{D}$-homotopic maps $\varphi, \psi: X \rightarrow$ $M-B$. Compositions $\varphi_{i_{j}}=\pi_{i_{j}} \circ \varphi$ and $\psi_{i_{j}}=\pi_{i_{j}} \circ \psi \operatorname{map} X$ into $N_{i_{j}}-B_{j}$, $j=1, \cdots, n$, and are $\mathscr{D}$-homotopic there. Let $r_{i_{j}}: N_{i_{j}}-B_{j} \rightarrow M_{i_{j}}$ $B_{j}$ be a right homotopy inverse to the inclusion $M_{i_{j}}-B_{j} \hookrightarrow N_{i_{j}}-$ $B_{j}$. Maps $r_{i_{j}} \circ \varphi_{i_{j}}$ and $r_{i_{j}} \circ \psi_{i_{j}}$ are $\mathscr{D}$-homotopic in $M_{i_{j}}-B_{j}$ and, therefore, they are homotopic in $M_{i_{i}}-A_{j}$ by the choice of sets $B_{j}$. It follows that $\phi_{i_{j}}$ and $\psi_{i_{j}}$ are homotopic in $N_{i_{j}}-A_{j}$. Since spaces $N_{i}$ are contractible, $\varphi_{i} \simeq \psi_{i}$, for all indices $i \neq i_{1}, \cdots, i_{n}$. Hence, $\varphi$ and $\psi$ are homotopic in $N-A$. As $X$ is globally left unstable in $N$ it is easy to see that $\varphi$ and $\psi$ are actually homotopic in $M-A$.

(5.7) THeOREM. Let $N$ be a compact space and let $X_{1} \supset X_{2} \supset \cdots$ be a decreasing sequence of its closed subsets. Suppose each $X_{i}$ is globally right unstable in $N$ and $X=\bigcap_{i>0} X_{i}$ is globally left unstable in $N$. If complements $M_{i}=N-X_{\imath}$ are $(\mathscr{C}, \mathscr{D})$-smooth at $\infty$, then $M=N-X$ is $(\mathscr{C}, \mathscr{D})$-smooth at $\infty$.

Proof. Let $A \subset M$ be a compact subset. The set $N-A$ is an open neighborhood of $X$ in $N$. Since $X$ is the intersection of $X_{i}^{\prime} \mathrm{s}$, we can find an integer $n \geqq 1$ such that $N-A$ is an open neighborhood of $X_{n}$. But $M_{n}=N-X_{n}$ is $(\mathscr{C}, \mathscr{D})$-smooth at $\infty$, so there is a compact $B \subset M_{n}$ with the property that every two $\mathscr{D}$-homotopic maps $\varphi, \psi: K \rightarrow M_{n}-B$ of $K \in \mathscr{C}$ into $M_{n}-B$ are homotopic in $M_{n}-A$.

In the commutative diagram of inclusions

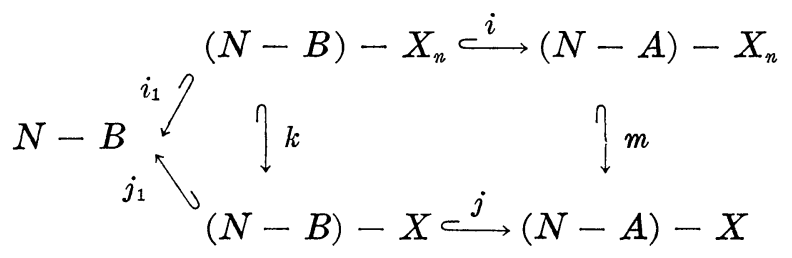

by assumption $i_{1}$ has a right homotopy inverse $i_{1}^{R}$ and $j_{1}$ has a left homotopy inverse $j_{1}^{L}$. Then $k \simeq j_{1}^{L} \circ i_{1}$ has a right homotopy inverse $k^{R}=i_{1}^{R} \circ j_{1}$. 
Assume $\varphi, \psi: K \rightarrow M-B$ are $\mathscr{D}$-homotopic maps of $K \in \mathscr{C}$ into $M-B$. Compositions $k^{R} \circ \varphi$ and $k^{R_{\circ}} \psi$ are $\mathscr{D}$-homotopic in $M_{n}-B$. Hence, $i \circ k^{R} \circ \varphi$ and $i \circ k^{R} \circ \psi$ are homotopic. Consequently, $m \circ i \circ k^{R} \circ \varphi \simeq$ $m \circ i \circ k^{R} \circ \psi$. But, $m \circ i \circ k^{R} \simeq j$ so that $j \circ \varphi \simeq j \circ \psi$, proving $M$ is ( $\mathscr{C}$, $\mathscr{D})$-smooth at $\infty$.

(5.8) Theorem. Let $\mathscr{C}$ and $\mathscr{D}$ be classes of compact spaces. If a locally compact space $M$ is the union of an increasing sequence of its $(\mathscr{C}, \mathscr{D})$-smooth at $\infty$ open subsets $M_{i}, i \geqq 0$, such that $M_{i+1}$

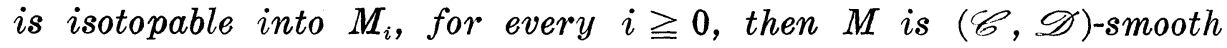
at $\infty$.

Proof. Let $A$ be a compact subset of $M$. Without loss of generality, we can assume $A \subset M_{0}$. As $M_{0}$ is $(\mathscr{C}, \mathscr{D})$-smooth at $\infty$, there is a compact $B \supset A$ in $M_{0}$ such that any two maps $\varphi^{\prime}, \psi^{\prime}: X \rightarrow$ $M_{0}-B$ of $X \in \mathscr{C}$ that are $\mathscr{D}$-homotopic in $M_{0}-B$ are homotopic in $M_{0}-A$.

Now, assume $\varphi, \dot{\psi}: X \rightarrow M-B$ are maps of $X \in \mathscr{C}$ into $M-B$ and suppose they are $\mathscr{D}$-homotopic in $M-B$. Let $n \geqq 0$ be an integer such that $\varphi(X) \cup \psi(X) \subset M_{n}$ and let $h_{t}: M_{n} \rightarrow M_{n}$ be an invertible isotopy keeping $B$ fixed with $h_{1}(\phi(X) \cup \psi(X)) \subset M_{0}-B$. If $Y \in \mathscr{D}$ and $\alpha: Y \rightarrow X$ is an arbitrary map, there is a homotopy $G: Y \times I \rightarrow M-B$ joining $\varphi \circ \alpha$ and $\psi \circ \alpha$. Since $Y$ is compact, there is an integer $m \geqq n$ such that $G(Y \times I) \subset M_{m}$. Let $h_{t}^{*}: M_{m} \rightarrow M_{m}$ be an invertible isotopy of $M_{m}$ which keeps $\varphi \circ \alpha(Y) \cup \psi \circ \alpha(Y) \cup B$ fixed and whose final stage throws $G(Y \times I)$ into $M_{n}$. It follows that $h_{1} \circ \varphi$ and $h_{1} \circ \psi$ are $\mathscr{D}$-homotopic maps into $M_{0}-B$. By the way $B$ was chosen, they are homotopic in $M_{0}-A$. Composing that homotopy with the isotopy $h_{t}^{-1}$ we see that $\varphi$ and $\psi$ are homotopic in $M-A$.

(5.9) Proposition. Let $N$ be the union of compacta $N_{1}$ and $N_{2}$ intersecting in a compact $A N R$ space $N_{0}$. Let $X \subset N$ be a closed connected subset and put $X_{i}=X \cap N_{i}$ and $M_{i}=N_{i}-X_{\imath}$, for $i=0$, 1,2. Suppose $M_{0}$ is contractible and $\mathscr{C} \mathscr{W}$-trivial at $\infty$ and $M_{0}$, $M_{1}$ and $M_{2}$ are one ended. If $M=N-X$ is $(\mathscr{C}, \mathscr{D})$-smooth at $\infty$, then both $M_{1}$ and $M_{2}$ are $(\mathscr{C}, \mathscr{D})$-smooth at ${ }^{\infty}$.

Proof. Consider an arbitrary compact subset $A_{1}$ of $M_{1}$. One easily constructs a proper retraction of $M_{2}$ onto $M_{0}$ (see [15, Theorem (4.5)] and, therefore, also a proper retraction $r: M \rightarrow M_{1}$. Hence, there is a compact subset $A$ of $M$ such that $r(M-A) \subset M_{1}-A_{1}$. Since $M$ is $(\mathscr{C}, \mathscr{D})$-smooth at $\infty$, there is a compact $B \subset M$ with the property that every two $\mathscr{D}$-homotopic maps $\varphi, \psi: X \rightarrow M-B$ 
of $X \in \mathscr{C}$ into $M-B$ are homotopic in $M-A$. Put $B_{1}=M_{1} \cap B$.

Let $\varphi, \psi: X \rightarrow M_{1}-B_{1}$ be $\mathscr{D}$-homotopic maps of $X \in \mathscr{C}$ into $M_{1}-B_{1}$. As $M_{1}-B_{1} \subset M-B$, the maps $\phi$ and $\psi$ are $\mathscr{D}$-homotopic in $M-B$ and, therefore, homotopic in $M-A$. Let a homotopy $h_{t}: X \rightarrow M-A$ join $\varphi$ and $\psi$. Clearly, $r \circ h_{t}: X \rightarrow M_{1}-A_{1}$ is a homotopy in $M_{1}-A_{1}$ between $\varphi$ and $\psi$.

(5.10) Definition. An end $e$ of a locally compact space $M$ is $(\mathscr{C}, \mathscr{D})$-smooth if for every open neighborhood $U$ of $e$ in $F M$, the Freudenthal compactification of $M$, there is smaller $V$ such that every two $\mathscr{D}$-homotopic maps $\varphi, \psi: X \rightarrow M \cap V$ of $X \in \mathscr{C}$ are homotopic in $U \cap M$.

(5.11) THEOREM. Let $\mathscr{C}$ be a component hereditary class of

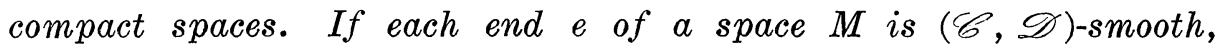
then $M$ is $(\mathscr{C}, \mathscr{D})$-smooth at $\infty$. The converse is also true without any assumptions about $\mathscr{C}$.

Proof. Let $A \subset M$ be any compact subset. Its complement $U=F M-A$ is an open neighborhood of each end $e \in E M$. Let $V_{e}$ be an open neighborhood of $e$ selected with respect to $U$ using $(\mathscr{C}, \mathscr{D})$-smoothness of $e$. Let $\mathscr{W}$ be a cover of $E M$ with disjoint open sets inscribed into $\left\{V_{e}\right\}_{e \in E M}$. Put $B=M-U\{W \mid W \in \mathscr{W}\}$.

Suppose $\varphi, \psi: X \rightarrow M-B$ are $\mathscr{D}$-homotopic maps of $X \in \mathscr{C}$ into $M-B$. Observe that, for each component $C$ of $X$, restrictions $\left.\varphi\right|_{C}$ and $\psi l_{C}$ are $\mathscr{D}$-homotopic maps of $C$ into some $V_{e}$. Since $C \in \mathscr{C}$, these restrictions are homotopic in $M-A$. This clearly implies that $\varphi$ and $\psi$ are homotopic in $M-A$.

Conversely, let $U$ be an open neighborhood of an end $e \in E M$. Let $U^{\prime} \subset U$ be a smaller neighborhood with $E M \cap U^{\prime}$ both closed and open in $E M$. Cover $E M-U^{\prime}$ with disjoint open sets $U_{1}, \cdots, U_{n}$ in $F M$. Put $A=M-\left(U^{\prime} \cup U_{1} \cup \cdots \cup U_{n}\right)$. Pick a compact $B \supset A$ applying $(\mathscr{C}, \mathscr{D})$-smoothness at $\infty$ of $M$. A required open neighborhood of $e$ is the set $V=(F M-B) \cap U^{\prime}$.

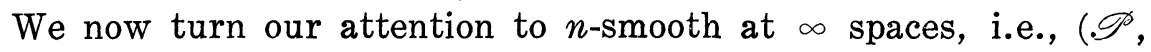
$\left.\mathscr{P}^{n}\right)$-smooth at $\infty$ spaces, and their ends. At first one is tempted to claim that an end [a] (as defined in [4]) of an ANR space $M$ is $n$-smooth if and only if Brown's group [4] $\underline{\pi}_{k}(M ; \underline{a})$ is trivial for all $k>n$. This statement is not true in general as the following examples show.

(5.12) ExAmPLE. Let $X$ be a celebrated compactum constructed by J. Taylor in [18]. Embed $X$ as a $Z$-set in the Hilbert cube $Q$ and let $M=Q-X$. By $[8,(3.9)(b)] M$ can not be $n$-smooth at $\infty$ 
for any $n \geqq 0$. It is well known (see [5]) that Brown's groups $\underline{\pi}_{k}(M ; \underline{a})$ of a unique end $[a]$ of $M$ are all trivial.

(5.13) EXAMPLE. The same properties as the previous example are also hold by the space $M$ obtained by glueing finite-dimensional spheres $\dot{S}^{1}, S^{2}, \ldots$ in such a way that two adjacent spheres meet in a single point.

The notation used in the statement and in the proof of (5.14) is taken from [4].

(5.14) Proposition. Let $M$ be an $A N R$ and let $\underline{a}$ be a germ of a representative of an end $[a]$ of $M$. If $M$ is n-smooth at $\infty$ and the group $\underline{\pi}_{1}(M ; \underline{a})$ is trivial, then the groups $\underline{\pi}_{k}(M ; \underline{a})$ are trivial, for all $k>n$.

Proof. Let $\varnothing=A_{1} \subset A_{2} \subset A_{3} \subset \cdots$ be an increasing sequence of compact subsets of $M$ such that $M=\bigcup_{i>0} A_{i}$ and any two $\mathscr{P}^{\text {n}}$-homotopic maps $\varphi, \psi: P \rightarrow M-A_{i+1}$ of a finite complex $P$ into $M-A_{i+1}$ are homotopic in $M-A_{i}, i>0$. Let $[f] \in \underline{\pi}_{k}(M ; \underline{a})$ be represented by a proper map $f:\left(\underline{S}^{k}, \underline{*}\right) \rightarrow(M, \underline{a})$. We can assume $f\left(S^{k} \times\{m\}\right) \subset$ $M-A_{m+3}$, for every $m \geqq 0$. Observe that, for every integer $m$, $f_{m}=\left.f\right|_{S^{k} \times\{m\}}$ is null-homotopic in $M-A_{m+2}$ since $f_{m}$ and a constant map of $S^{k} \times\{m\}$ into $M-A_{m+3}$ are $\mathscr{P}^{k-1}$-homotopic. The point $(*, m)$ will under that null-homotopy, denoted $h_{m, t}$, be carried along an arc in $M-A_{m+2}$ from the point $f(*, m)=h_{m, 0}(*, m)$ to the point $h_{m, 1}(*, m)$. Define a map $\lambda_{m}$ on the boundary of a 2-cell $\{(*, m)\} \times I \times I$ into $M-A_{m+2}$ as $h_{m, t}$ on $\{(*, m)\} \times I \times\{0\}$, as $h_{m, 1-t}$ on $\{(*, m)\} \times\{1\} \times I$, and as $h_{m, 0}$ on $\{(*, m)\} \times(\{0\} \times I \cup I \times\{1\})$. Maps $\lambda_{m}$ in an obvious way give us an element of $\underline{\pi}_{1}(M ; \underline{a})$. Since we assumed that this group is trivial, we conclude that each map $\lambda_{m}$ can be, without loss of generality, extended to a map $L_{m}:\{(*, m)\} \times I \times I \rightarrow M-A_{m+1}$. With that observation, applying homotopy extension theorem, one easily proves that $f_{m}$ is in $M-A_{m+1}$ null-homotopic relative to the point $(*, m)$. Hence, $[f]$ is the trivial element of $\underline{\pi}_{k}(M ; \underline{a})$.

(5.15) Corollary. Let $M$ be an $\left\{S^{1}\right\}$-trivial at $\infty$ ANR space. If $M$ is $n$-smooth at $\infty$, then for each a representing an arbitrary end $[a]$, the groups $\underline{\pi}_{k}(M ; \underline{a})$ are trivial, $k>n$.

Proof. Follows from (5.14) because an ANR space $M$ is $\left\{S^{1}\right\}$ trivial at $\infty$ if and only if groups $\underline{\pi}_{1}(M ; \underline{a})$ are all trivial. The last statement is proved by a method used in the proof of (5.14).

Related to (5.14) and (5.15) is also the following observation. 
(5.16) Proposition. If $M$ is an n-trivial at $\infty$ and $n$-smooth at $\infty$ locally compact space, then $M$ is trivial at $\infty$.

Proof. Let $A$ be a compact subset of $M$. Select a compact set $B^{\prime}$ with respect to $A$ using $n$-smoothness at $\infty$ of $M$. Then take a compact $B$ with respect to $B^{\prime}$ applying $n$-triviality at $\infty$ of $M$.

Let $\varphi: P \rightarrow M-B$ be a map of a finite complex $P$ into a component of $M-B$. The restriction $\left.\varphi\right|_{P^{n}}: P^{n} \rightarrow M-B$ of $\varphi$ onto the $n$-skeleton of $P$ is null-homotopic in $M-B^{\prime}$. Hence, there is a constant map $\psi: P \rightarrow M-B^{\prime}$ such that $\varphi$ and $\psi$ are $\mathscr{P}^{n}$-homotopic in $M-B^{\prime}$. The choice of $B^{\prime}$ implies $\varphi \simeq \psi$ in $M-A$. Thus $\varphi$ is null-homotopic in $M-A$.

At present we can prove only the following partial converse of (5.15) under rather strong movability type conditions on the space $M$.

(5.17) THEOREM. Suppose $M$ is calm at $\infty$, movable at $\infty$, and $\left\{S^{k}\right\}$-trivial at $\infty$, for every $k>n \geqq 0$. Then $M$ is $n$-smooth at $\infty$.

Proof. Let $A$ be a compact subset of $M$. Pick a compact set $C$ containing $A$ using calmness at $\infty$ of $M$ and a compact $B \supset C$ using movability at $\infty$ of $M$. We claim that $B$ is a compact set satisfying (2.4) for $\mathscr{C}=\mathscr{P}^{\circ}$ and $\mathscr{D}=\mathscr{P}^{n}$.

Indeed, let $\varphi, \psi: P \rightarrow M-B$ be any maps of a finite polyhedron $P$ into $M-B$ and assume that their restrictions $\varphi^{n}, \psi^{n}$ on the $n$ skeleton of $P$ are homotopic. Let $k=\operatorname{dim} P$. Without loss of generality we can assume $k>n$. Let compact sets $D_{n+1} \supset D_{n+2} \supset \ldots$ $\supset D_{k-1} \supset D_{k} \supset D_{k+1}=B$ be chosen in such a way that every singular $i$-sphere in $M-D_{i}$ is null-homotopic in $M-D_{i+1}, i=n+1, n+2$, $\cdots, k$. Let $D_{n+1}^{*}$ be picked with respect to $C$ and $D_{n+1}$ using calmness at $\infty$ of $M$.

By the choice of $B$ we see that maps $\varphi$ and $\psi$ are in $M-C$ homotopic to maps $\varphi^{\prime}$ and $\psi^{\prime}$, respectively, mapping $P$ into $M-$ $D_{n+1}^{*}$. Note that $\left(\varphi^{\prime}\right)^{n}$ and $\left(\psi^{\prime}\right)^{n}$ are homotopic in $M-C$. The way $D_{n+1}^{*}$ and $C$ were chosen gives $\left(\varphi^{\prime}\right)^{n} \simeq\left(\psi^{\prime}\right)^{n}$ in $M-D_{n+1}$. Now, the property of $D_{n+1}$ implies $\left(\varphi^{\prime}\right)^{n+1} \simeq\left(\psi^{\prime}\right)^{n+1}$ in $M-D_{n+2}$. Hence, $\left(\varphi^{\prime}\right)^{n+2}$ and $\mid\left(\psi^{\prime}\right)^{n+2}$ are homotopic in $M-D_{n+3}$. Continuing in this way we see that $\varphi^{\prime}=\left(\varphi^{\prime}\right)^{k}$ and $\psi^{\prime}=\left(\psi^{\prime}\right)^{k}$ are homotopic in $M-D_{k+1}=M-B$. Finally, it follows that $\varphi$ is homotopic to $\psi$ in $M-C \subset M-A$.

(5.18) KEMARK. The assumption that $M$ is movable at $\infty$ can not be dropped out in (5.17) since the space $M$ from (5.13) is calm at $\infty,\left\{S^{k}\right\}$-trivial at $\infty$, for every $k \geqq 0$, nonmovable at $\infty$ and 
not $n$-smooth at $\infty$ for any $n \geqq 0$.

6. $(\mathscr{C}, \mathscr{D})$-smooth compacta. In this section, by looking at complements of $Z$-sets in the Hilbert cube, we define a class of $(\mathscr{C}, \mathscr{D})$-smooth compacta in much the same way as we defined $\mathscr{C}$-calm compacta starting from the notion of a $\mathscr{C}$-calm at $\infty$ space. Then we state a few results concerning this class and give some examples. The proofs are omitted because in method they are the same as the proofs given in $\S 4$ or they can be proved rather easily from the corresponding results in $\S 5$.

(6.1) Definition. A compact metric space $X$ is $(\mathscr{C}, \mathscr{D})$-smooth provided there is a $Z$-set copy $X^{\prime}$ of $X$ in $Q$ such that $M=Q-X^{\prime}$ is $(\mathscr{C}, \mathscr{D})$-smooth at $\infty$. Compacta $\left(\mathscr{P}, \mathscr{P}^{n}\right)$-smooth are called $n$ smooth.

(6.2) ExAmple. A compactum $X$ is $O$-smooth if and only if every component of $X$ has trivial shape.

(6.3) THEOREM. The following statements about a compactum $X$ are equivalent.

(i) $X$ is $(\mathscr{C}, \mathscr{D})$-smooth.

(ii) If $X^{\prime}$ is a $Z$-set in an $A N R$ space $N$ and is homeomorphic to $X$, then $N-X^{\prime}$ is $(\mathscr{C}, \mathscr{D})$-smooth at $\infty$.

(iii) If $X^{\prime}$ is a $Z$-set copy of $X$ in an $A N R N$, then $X^{\prime} \in(\mathscr{C}$, $\mathscr{D})(N)$, i.e., for every open neighborhood $U$ of $X^{\prime}$ in $N$ there is a smaller $V$ such that every two $\mathscr{D}$-homotopic maps $\varphi, \psi: K \rightarrow V$ of $K \in \mathscr{C}$ into $V$ are homotopic $U$. $(N)$.

(iv) If $X$ is a closed subset of an $A N R N$, then $X \in(\mathscr{C}, \mathscr{D})$

(v) If $X=\lim \sigma$, where $\sigma=\left\{X_{i}, f_{i^{\prime}}^{i}\right\}_{i^{\prime} \geqq i>0}$ is an inverse sequence of ANR's, then the infinite mapping cylinder $\operatorname{Map}(\sigma)$ of $\sigma$ is ( $\mathscr{B}$ D) -calm at $\infty$.

(vi) Assume $X=\lim \sigma$, where $\sigma=\left\{X_{i}, f_{i^{\prime}}^{i}\right\}_{i^{\prime} \geqq i>0}$ is an inverse sequence of ANR's. Then for every index $i>0$ there is $j \geqq i$ such that, for every two $\mathscr{D}$-homotopic maps $\varphi, \psi: K \rightarrow X_{j}$ of $K \in \mathscr{C}$ into $X_{j}$, compositions $f_{j}^{i} \circ \varphi$ and $f_{j}^{i} \circ$ ir are homotopic.

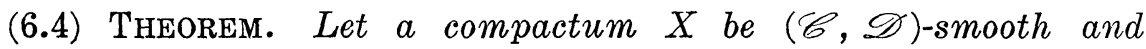
assume $X$ quasi-dominates a compactum $Y$ [2]. Then $Y$ is also $(\mathscr{B}, \mathscr{D})$-smooth.

(6.5) Proposition. Assume $\mathscr{C}$ and $\mathscr{C}^{\prime}$ are classes of spaces with homotopy types of ANR's. 
(a) If a compactum $X$ is $(\mathscr{C}, \mathscr{D})$-smooth and a class $\mathscr{D}$ is shape dominated by a class $\mathscr{D}^{\prime}$, then $X$ is $\left(\mathscr{C}, \mathscr{D}^{\prime}\right)$-smooth.

(b) If a compactum $X$ is $(\mathscr{C}, \mathscr{D})$-smooth and $\mathscr{C}^{\prime}$ is homotopy dominated by $\mathscr{C}$, then $X$ is also $\left(\mathscr{C}^{\prime}, \mathscr{D}\right)$-smooth.

(6.6) THEOREM. The product $X=\Pi_{i>0} X_{i}$ of countably many compacta $X_{i}$ is $(\mathscr{C}, \mathscr{D})$-smooth if and only if each $X_{i}$ is $(\mathscr{C}, \mathscr{D})$ smooth.

(6.7) THEOREM. The intersection $X$ of any decreasing sequence $X_{1} \supset X_{2} \supset \cdots$ of $(\mathscr{C}, \mathscr{D})$-smooth compacta is $(\mathscr{C}, \mathscr{D})$-smooth.

(6.8) Proposition. Let $X$ be the union of compacta $X_{1}$ and $X_{2}$ intersecting in a compactum of trivial shape $X_{0}$. If $X$ is $(\mathscr{C}, \mathscr{D})$ smooth, then both $X_{1}$ and $X_{2}$ are $(\mathscr{C}, \mathscr{D})$-smooth.

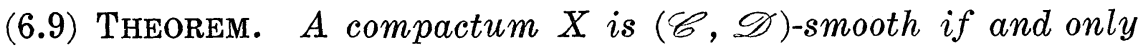
if each component of $X$ is $(\mathscr{C}, \mathscr{D})$-smooth.

(6.10) ExAMPLE. Taylor's space $X$ [18] is not n-smooth for any $n \geqq 0$.

(6.11) Proposition. Let $X$ be an approximatively 1-connected continum. If $X$ is $n$-smooth, then pro-groups $\pi_{k}(X)$ are trivial for all $k>n$.

(6.12) Proposition. A compactum $X$ has trivial shape if an only if $X$ is approximatively n-connected and n-smooth.

(6.13) Theorem. Suppose $X$ is a movable, calm, and approxi. matively k-connected, for all $k>n$, compactum. Then $X$ is $n$ smooth.

(6.14) Example. The 1-sphere $S^{1}$ is 1 -smooth.

\section{REFERENCES}

1. B. J. Ball, Proper shape retracts, Fund. Math., 89 (1975), 177-189.

2. K. Borsuk, Theory of shape, Monografie Matematyczne 59, Warszawa, 1975.

3. - Theory of retracts, Monografie Matematyczne 44, Warszawa, 1967.

4. E. M. Brown, Proper homotopy theory in simplicial complexes, Lecture Notes in Math., Vol. 375, Springer, New York, 1972.

5. Z. Čerin, n-docility at $\infty$ and compactifications of $L C^{n}$ spaces, Glasnik Mat., 12 (1977), 161-174.

6. - Homotopy properties of locally compact spaces at infinity-triviality and movability, Glasnik Mat. (to appear). 
7. Z. Čerin, Locally compact spaces $\mathscr{C}$-tame at infinity, Publ. Inst. Math., 22 (36) (1977), 49-59.

8. - Homotopy at infinity of proper maps, Glasnik Mat., 13 (1978). 135-154.

9. - Infinite dimensional topology and the theory of shape, Doctoral Dissertation, LSU, Baton Rouge, 1975.

10. T. A. Chapman, On some applications of infinite-dimensional manifolds to the theory of shape, Fund. Math., 76 (1972), 181-193.

11. L Lectures on Hilbert cube manifolds, Regional conference series in mathematics, No. 28, Amer. Math. Soc., Providence, 1976.

12. T. A. Chapman and L. C. Siebenmann, Finding a boundary for a Hilbert cube manifold, Acta Math., 137 (1976), 171-208.

13. G. Kozlowski, Images of ANR's, Trans. Amer. Math. Soc., (to appear).

14. -, (in preparation).

15. R. B. Sher, Docility at infinity and compactifications of ANR's, Trans. Amer. Math. Soc., 221 (1976), 213-224.

16. L. C. Siebenmann, L. Guillou, and H. Hähl, Les voisinages ouverts réguliers, Ann. Scient. Èc. Norm. Sup., 6 (1973), 253-293.

17. Les voisinages ouverts réguliers: Criteres homotopiques d'existence, ibid. 7 (1974), 431-462.

18. J. L. Taylor, A counterexample in shape theory, Bull. Amer. Math. Soc., 81 (1975), 629-632.

Received June 1977.

UNIVERSITY OF ZAGREB

41001 ZAGREB, p. p. 187

YUgosLAVIA 



\section{PACIFIC JOURNAL OF MATHEMATICS}

EDITORS

RICHARD ARENS (Managing Editor)

University of California

Los Angeles, California 90024

C. W. Curtis

University of Oregon

Eugene, OR 97403

C. C. MOORE

University of California

Berkeley, CA 94720
J. DUGUNDJI

Department of Mathematics University of Southern Californı Los Angeles, California 90007

R. Finn and J. Milgram Stanford University Stanford, California 94305

\section{ASSOCIATE EDITORS}

E. F. BECKENBACH

B. H. NEUMANN

F. WOLF

K. YoSHIDA

\section{SUPPORTING INSTITUTIONS}

UNIVERSITY OF BRITISH COLUMBIA
CALIFORNIA INSTITUTE OF TECHNOLOGY
UNIVERSITY OF CALIFORNIA
MONTANA STATE UNIVERSITY
UNIVERSITY OF NEVADA, RENO
NEW MEXICO STATE UNIVERSITY
OREGON STATE UNIVERSITY
UNIVERSITY OF OREGON

UNIVERSITY OF SOUTHERNEALIFORNIA STANFORD UNIVERSITY UNIVERSITY OF HAWAII UNIVERSITY OF TOKYO UNIVERSITY OF UTAH WASHINGTON STATE UNIVERSITY UNIVERSITY OF WASHINGTON 


\section{Pacific Journal of Mathematics \\ Vol. 79 , No. 1 \\ May, 1978}

Teófilo Abuabara, A remark on infinitely nuclearly differentiable

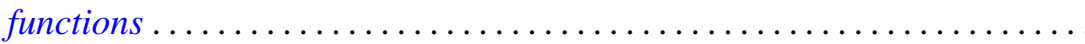

David Fenimore Anderson, Projective modules over subrings of $k[X, Y]$

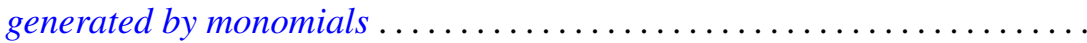

Joseph Barback and Thomas Graham McLaughlin, On the intersection of

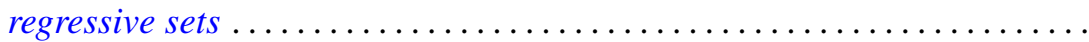

Murray Bell, John Norman Ginsburg and R. Grant Woods, Cardinal inequalities for topological spaces involving the weak Lindelof

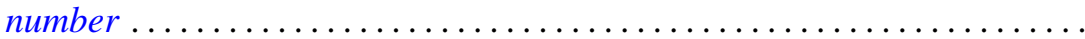

Laurence Richard Boxer, The space of ANRs of a closed surface ............

Zvonko Cerin, Homotopy properties of locally compact spaces at

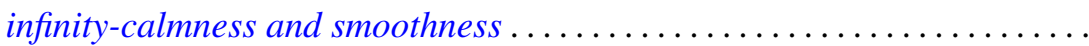

Isidor Fleischer and Ivo G. Rosenberg, The Galois connection between partial functions and relations..................................

John R. Giles, David Allan Gregory and Brailey Sims, Geometrical implications of upper semi-continuity of the duality mapping on a Banach

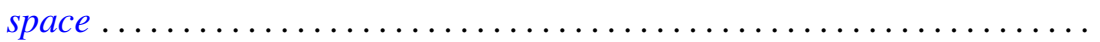

Troy Lee Hicks, Fixed-point theorems in locally convex spaces ............ Hugo Junghenn, Almost periodic functions on semidirect products of transformation semigroups ........................

Victor Kaftal, On the theory of compact operators in von Neumann algebras. II . . . .

Haynes Miller, A spectral sequence for the homology of an infinite

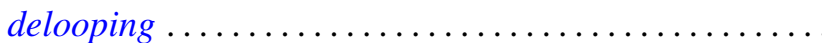

Sanford S. Miller, Petru T. Mocanu and Maxwell O. Reade, Starlike integral operators...

Stanley Stephen Page, Regular FPF rings ...............

Ghan Shyam Pandey, Multipliers for C, 1 summability of Fourier series ...

Shigeo Segawa, Bounded analytic functions on unbounded covering surfaces...

Steven Eugene Shreve, Probability measures and the C-sets of

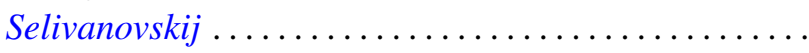

Tor Skjelbred, Combinatorial geometry and actions of compact Lie groups....

Alan Sloan, A note on exponentials of distributions.

Colin Eric Sutherland, Type analysis of the regular representation of a nonunimodular group.

Mark Phillip Thomas, Algebra homomorphisms and the functional

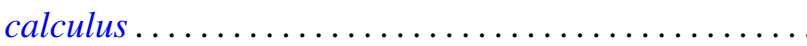

Sergio Eduardo Zarantonello, A representation of $H^{p}$-functions with

$0<p<\infty$. 